\title{
't Hooft Tensor for generic Gauge Groups
}

\author{
A. Di Giacomo*a L. Lepori ${ }^{\mathrm{b}}$ F.Pucci ${ }^{\mathrm{c}}$

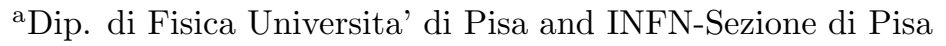 \\ Largo B. Pontecorvo 3, 56127 Pisa, Italy \\ ${ }^{\mathrm{b}}$ International School for Advanced Studies (SISSA) and INFN-Sezione di Trieste \\ Via Beirut 2-4, 34014 Trieste, Italy
}

'Dip. Fisica Universita' di Firenze and INFN-Sezione di Firenze

Via G. Sansone 1, 50019 Sesto Fiorentino, Italy

\begin{abstract}
We study monopoles in gauge theories with generic gauge group. Magnetic charges are in one-to-one correspondence with the second homotopy classes at spatial infinity $\left(\Pi_{2}\right)$, which are therefore identified by the 't Hooft tensor. We determine the 't Hooft tensor in the general case. These issues are relevant to the understanding of Color Confinement.
\end{abstract}

\section{INTRODUCTION}

No quark has ever been observed in Nature. The ratio of the abundance of quarks in ordinary matter $n_{q}$ to the abundance of protons $n_{p}$ has an experimental upper limit $\frac{n_{q}}{n_{p}} \leq 10^{-27}$, to be compared to the expectation in the Standard Cosmological Model [1] $\frac{n_{q}}{n_{p}} \approx 10^{-12}$. The inhibition factor which quantifies confinement is $\approx 10^{-15}$.

A similar factor limits the production of quarks in high energy reactions. The cross section for inclusive production of quarks plus antiquarks has an experimental upper limit $\sigma_{q} \equiv \sigma(p+p \rightarrow$ $q(\bar{q})+X) \leq 10^{-40} \mathrm{~cm}^{2}$, to be compared to the perturbative estimate $\sigma_{q} \approx \sigma_{T O T} \approx 10^{-25} \mathrm{~cm}^{2}$ Again an inhibition factor of $\approx 10^{-15}$.

The natural explanation is that $n_{q}=0$ and $\sigma_{q}=0$ protected by some symmetry: this is similar to what happens in ordinary superconductivity, where the resistivity is constrained by experiment to be a very small fraction of that of the normal metal. The resistivity of the superconductor is strictly zero, due to the Higgs breaking of electric charge $U(1)$ symmetry which is restored in the normal phase.

If this is true the deconfining transition is a

${ }^{*}$ Presented the talk change of symmetry, i.e. an order-disorder transition, and can not be a crossover. In particular an order parameter must exist which unambiguously defines confinement versus deconfinement.

Two main questions follow immediately:

a) What is the symmetry related to confinement? Color is an exact symmetry, both in confined and in deconfined phase, and therefore can not be our symmetry. We need an extra symmetry besides color.

b) There is no direct experimental observation yet of the deconfining transition, but it has been observed in lattice simulations. Are observations on the lattice compatible with an order disorder transition?

The question b) is still open [2, 3] and will not be discussed here. We shall instead address here the question a).

In the absence of quarks the action is blind to the center $Z_{3}$ of the gauge group. The theory can be however formulated in terms of $3 \times 3$ matrices of the fundamental representation of the gauge group, to allow the introduction of static external quarks. $Z_{3}$ is then an extra symmetry, and the Polyakov line an order parameter, detecting confinement and deconfinement. In presence of dynamical quarks, as in Nature, $Z_{3}$ is explicitly broken and therefore can not be the symmetry we look for. An extra symmetry can only be pro- 
vided by dual degrees of freedom, i.e. by infrared modes with non trivial spatial homotopy, or by global properties of the field configurations, which exist besides local gauge symmetry.

The natural excitations are vortices in $(2+1)$ dimensional QCD, where the surface at spatial infinity is a circle and the homotopy $\Pi_{1}$, they are monopoles in the physically realistic case of QCD in $(3+1)$ dimensions, where the surface at spatial infinity is that of a sphere and the homotopy $\Pi_{2}$.

\section{MONOPOLES}

The prototype monopole configuration in gauge theories is that of ref [5] [6. It is a soliton solution in the Higgs broken phase of an $\mathrm{SO}(3)$ gauge theory coupled to a Higgs field in the adjoint representation.

$L=-\frac{1}{4} \vec{G}_{\mu \nu} \vec{G}_{\mu \nu}+\left(D_{\mu} \vec{\Phi}\right)^{2}-V\left(\Phi^{2}\right)$

In the "hedgehog" gauge the soliton solution is

$\phi^{a}=f(r) \frac{r^{a}}{r} \quad A_{i}^{a}=g(r) \epsilon_{a i j} \frac{r^{i}}{r^{2}}$

$f(r)$ and $g(r)$ are $\approx 1$ outside a radius which is determined by the Higgs vev $\langle\Phi\rangle$. $\phi^{a}$ has been normalized to be 1 at $x \rightarrow \infty$. The solution is a non trivial mapping of the 2-dim sphere at infinity $S_{2}$ onto $S O(3) / U(1)$. Going to the unitary gauge where $\vec{\phi}=(0,0,1)$, a line-like singularity appears starting from $\vec{x}=0$. At large distances the abelian field strength of the residual $U(1)$ gauge symmetry in the unitary gauge

$F_{\mu \nu}^{3}=\partial_{\mu} A_{\nu}^{3}-\partial_{\nu} A_{\mu}^{3}$

is for the soliton solutions

$E_{i} \equiv F_{0 i}^{3}=0$

$\vec{H}=\frac{1}{g} \frac{\vec{r}}{4 \pi r^{3}}+$ Dirac - string

with $H_{i}=\frac{1}{2} \epsilon_{i j k} F_{j k}^{3}$. If the string is invisible, as happens in a compact formulation like lattice, there is a violation of Bianchi identities

$\vec{\nabla} \vec{H}=\frac{1}{g} \delta^{3}(\vec{x})$
More formally, one can define a gauge invariant tensor [5], the 't Hooft tensor $F_{\mu \nu}$, which is equal to the abelian field strength $F_{\mu \nu}^{3}$ of eq(3) in the unitary gauge.

$F_{\mu \nu} \equiv \vec{\Phi} \vec{G}_{\mu \nu}-\frac{1}{g} \vec{\Phi}\left(\overrightarrow{D_{\mu}} \Phi \wedge \overrightarrow{D_{\nu}} \Phi\right)$

One can show that

$F_{\mu \nu}=\partial_{\mu}\left(\vec{\phi} \overrightarrow{A_{\nu}}\right)-\partial_{\nu}\left(\vec{\phi} \vec{A}_{\mu}\right)-\frac{1}{g} \vec{\phi}\left(\partial_{\mu} \vec{\phi} \wedge \partial_{\nu} \vec{\phi}\right)$

which is exactly $F_{\mu \nu}^{3}$ of eq(3) in the unitary gauge where $\partial_{\mu} \vec{\phi}=0$.

If we denote by $\tilde{F}_{\mu \nu}=\frac{1}{2} \epsilon_{\mu \nu \rho \sigma} F_{\rho \sigma}$ the dual of $F_{\mu \nu}$, we can define a magnetic current $j_{\nu}$ as

$j_{\nu} \equiv \partial_{\mu} \tilde{F}_{\mu \nu}$

A non zero $j_{\nu}$ indicates violation of Bianchi identities, i.e. presence of magnetic charges. Whatever the lagrangian is, the antisymmetry of $F_{\mu \nu}$ implies

$\partial_{\nu} j_{\nu}=0$

This defines the dual symmetry, which is nothing but conservation of magnetic charge. Formally the values of the charge are the elements of the homotopy group $\Pi_{2}(S O(3) / U(1))$ which is easily computed to be $Z / Z_{2}$. Only even valued magnetic charges are allowed.

A real Higgs breaking is only needed if one wants monopoles as solitons. The field configurations can in any case be classified by their homotopy and the dual current can be defined anyhow. Also the Higgs field is not needed: any operator $\Phi$ in the adjoint representation can be used to define $F_{\mu \nu}$ and the dual symmetry. Monopole singularities will be located at the zeroes of $\Phi$ which are a non dense set [7] and the particular choice of $\Phi$ becomes irrelevant if one defines the field theory in space-time with a discrete set of singularities excluded [8]. In particular any operator $\mu$ which creates a monopole 9] 10, 11] adds one extra singularity to field configurations, i.e. a monopole, for whatever choice of $\Phi$. Its vev $\langle\mu\rangle$ detects dual superconductivity of the vacuum and color confinement, since $\langle\mu\rangle=0$ when the vacuum state has definite magnetic charge, i.e. 
when the $U(1)$ symmetry is realized $a$ la Wigner (deconfined phase). If, instead, $\langle\mu\rangle \neq 0$ the vacuum is a Bogoliubov superposition of states with different magnetic charge, i.e. a dual superconductor. Since $\mu$ creates a monopole for whatever choice of the operator $\Phi$ the statement is independent on it.

\section{EXTENSION TO GENERIC GAUGE GROUPS 12}

The gauge group of strong interactions in Nature is color $S U(3)$. However, to get insight into the mechanism of confinement, theories with different gauge groups can be studied. For example, to clarify the role played by center vortices a study of confinement for gauge groups which have trivial center, and hence no vortices can be useful [13]: this is the motivation for what follows. To identify field configurations with non trivial $\Pi_{2}$, one needs an $S U(2)$ subgroup of the gauge group $G$ to be broken to $U(1)$, e.g. to its third generator. The $S U(2)$ subgroups of $G$ are easily visualized by looking at the Lie algebra $g . g$ is spanned by the Cartan commuting operators $H_{i}$ $(i=1, . ., r)$, with $r$ the rank of the group, and by $E_{ \pm \vec{\alpha}}$, with $\vec{\alpha}$ the roots of $g$. In the standard notation

$$
\begin{aligned}
& {\left[H_{i}, H_{j}\right]=0} \\
& {\left[H_{i}, E_{ \pm \vec{\alpha}}\right]= \pm \vec{\alpha} E_{ \pm \vec{\alpha}}} \\
& {\left[E_{\vec{\alpha}}, E_{\vec{\beta}}\right]=N_{\vec{\alpha} \vec{\beta}} E_{\vec{\alpha}+\vec{\beta}}} \\
& {\left[E_{\vec{\alpha}}, E_{-\vec{\alpha}}\right]=(\vec{\alpha} \vec{H})}
\end{aligned}
$$

To each root $\vec{\alpha}$ an $S U(2)$ subgroup is associated as can be seen operating a trivial renormalization of the generators:

$$
\begin{aligned}
T_{ \pm}^{\alpha} & \equiv \sqrt{\frac{2}{(\vec{\alpha} \vec{\alpha})}} E_{ \pm} \vec{\alpha} \\
T_{3}^{\alpha} & \equiv \frac{(\vec{\alpha} \vec{H})}{(\vec{\alpha} \vec{\alpha})}
\end{aligned}
$$

obeying to the commutation rules:

$$
\begin{aligned}
& {\left[T_{3}^{\alpha}, T_{ \pm}^{\alpha}\right]= \pm T_{ \pm}^{\alpha}} \\
& {\left[T_{+}^{\alpha}, T_{-}^{\alpha}\right]=2 T_{3}^{\alpha}}
\end{aligned}
$$

A root $\vec{\alpha}$ is called positive if its first non zero component is positive: either $\vec{\alpha}$ or $-\vec{\alpha}$ is a positive root. A positive root is called simple if it cannot be written as a sum of two positive roots. Any positive root can be made simple by a suitable (Weyl) transformation of the group. Without loss of generality, we can then only consider the monopoles associated to simple roots. Simple roots are represented by little circles in the Dynkin diagram of the algebra.

Let $\phi^{a}$ be the fundamental weight corresponding to the simple root $\vec{a}$. Since $\phi^{a}$ commutes with the generators corresponding to all the other simple roots different from $\vec{a}$, the invariance group of $\phi^{a}$ is a group having as Dynkin diagram the diagram obtained by erasing the little circle corresponding to the root $\vec{a}$ times the $U(1)$ generated by $\phi^{a}$ itself 12 .

For gauge group $S U(N)$ there are $(N-1)$ simple roots and the Dynkin diagram has the form

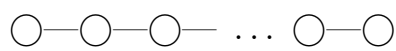

with $(N-1)$ simple roots of equal length $\vec{a}^{i}, i=$ $1, \ldots, N-1$. By erasing the i-th simple root one obtains the Lie algebra of the invariance group, which is that of $S U(i) \otimes S U(N-i) \otimes U(1)$. In considering global features the knowledge of Lie algebra is not sufficient to identify the real invariance group. For example, in the case of $S U(N)$ the elements of the center of $S U(i)$ and $S U(N-i)$ can also be elements of the $U(1)$ group generated by $\phi^{a}$ so that, when embedding in $S U(N)$, there is a non trivial kernel, which is composed by the set of elements of $S U(i) \otimes S U(N-i) \otimes U(1)$ which are mapped to the identity of $S U(N)$. By carefully taking this into account one realizes that topologies are in one-to-one correspondence with magnetic charges. This is not only true for $S U(N)$ but also for any compact simple group, including the exceptional groups 12 .

For each simple root $\vec{a}$ a 't Hooft tensor $F_{\mu \nu}^{a}$ can be defined as the abelian field strength in the unitary gauge and with it also a conserved current $j_{\nu}^{a}=\partial_{\mu} \widetilde{F}_{\mu \nu}^{a}$

$\partial_{\nu} j_{\nu}^{a}=0$

The corresponding conserved charge is the magnetic charge $Q^{a}$. In the deconfined phase the 
operator $Q^{a}$ is well defined and magnetic charge is superselected. In the confined phase, instead, the corresponding gauge symmetry is broken a la Higgs, and the vacuum is a superposition of states with different magnetic charge. A set of operators $\mu^{a}$ can be defined, which carry non zero $Q^{a}$ magnetic charge, and in terms of them $r$ order parameters $\left\langle\mu^{a}\right\rangle$ for detecting confinement. In the deconfined phase magnetic charges are superselected and $\left\langle\mu^{a}\right\rangle=0$. If $\left\langle\mu^{a}\right\rangle \neq 0$ the symmetry is Higgs broken, the vacuum is a dual superconductor and there is confinement.

\section{THE 'T HOOFT TENSOR}

The 't Hooft tensor can be given as an explicit gauge invariant form for any gauge group 12. This is a gauge invariant tensor equal to the residual abelian field strength in the unitary gauge. The magnetic field coupled to the i-th magnetic charge is that of the residual gauge group $U(1)^{i}$ generated by $T_{3}^{i}$. The e.m. field $A_{\mu}^{i}$ is defined in terms of the gauge field $A_{\mu}^{\prime}$ in the unitary gauge as:

$A_{\mu}^{i}=\operatorname{Tr}\left(\phi_{0}^{i} A_{\mu}^{\prime}\right)$

$\phi_{0}^{i}=\mu^{i}$, the fundamental weight $(\mathrm{i}=1, \ldots, r)$, identifies the monopole species. If $b(x)$ is the transformation bringing to a generic gauge and $A_{\mu}$ the transformed gauge field [14]

$$
\left\{\begin{array}{c}
A_{\mu}^{\prime}=b A_{\mu} b^{-1}-\frac{i}{g}\left(\partial_{\mu} b\right) b^{-1} \\
\phi_{0}^{i}=b \phi^{i} b^{-1}
\end{array}\right.
$$

the e.m. field is given by:

$A_{\mu}^{i}=\operatorname{Tr}\left(\phi^{i}\left(A_{\mu}+\Omega_{\mu}\right)\right)$

where $\Omega_{\mu}=-\frac{i}{g} b^{-1} \partial_{\mu} b$. We can rewrite the abelian field strength as

$F_{\mu \nu}^{i}=\operatorname{Tr}\left(\phi^{i} G_{\mu \nu}\right)+i g \operatorname{Tr}\left(\phi^{i}\left[A_{\mu}+\Omega_{\mu}, A_{\nu}+\Omega_{\nu}\right]\right)$

$F_{\mu \nu}^{i}$ can computed [12] starting from the observation that the ciclycity of the trace implies that only the part of $V_{\mu} \equiv A_{\mu}+\Omega_{\mu}$, which does not belong to the invariance group of $\phi^{i}$, contributes. Indeed

$\operatorname{Tr}\left(\phi^{i}\left[V_{\mu}, V_{\nu}\right]\right)=\operatorname{Tr}\left(V_{\nu}\left[\phi^{i}, V_{\mu}\right]\right)=\operatorname{Tr}\left(V_{\mu}\left[V_{\nu}, \phi^{i}\right]\right)(20)$
To compute the second term in eq. (19), it proves convenient to introduce a projector $P^{i}$ on the complement of the invariance algebra of $\phi^{i}$, and to write Eq.(19) in the form

$F_{\mu \nu}^{i}=\operatorname{Tr}\left(\phi^{i} G_{\mu \nu}\right)+i g \operatorname{Tr}\left(\phi^{i}\left[P^{i}\left(A_{\mu}+\Omega_{\mu}\right), A_{\nu}+\Omega_{\nu}\right]\right)$

It is proved in ref[12] that projection on the complement $P^{i} V_{\mu}$ is given by

$P^{i} V_{\mu}=1-\prod_{\vec{\alpha}}^{\prime}\left(1-\frac{\left[\phi^{i},\left[\phi^{i},\right]\right]}{\left(\vec{c}^{i} \cdot \vec{\alpha}\right)^{2}}\right) V_{\mu}$

where $\left[\phi^{i},\right] V_{\mu}=\left[\phi, V_{\mu}\right]$, the product $\prod_{\vec{\alpha}}^{\prime}$ runs on the roots $\vec{\alpha}$ such that $\vec{c}^{i} \cdot \vec{\alpha} \neq 0$ and only one representative of the set of the roots having the same value of $\vec{c}^{i} \cdot \vec{\alpha}$ is taken. In order to simplify the notation we denote by $\lambda_{I}^{i}$ the different non zero values which $\left(\vec{c}^{i} \cdot \vec{\alpha}\right)^{2}$ can assume and rewrite $P^{i} V_{\mu}$ as

$P^{i} V_{\mu}=1-\prod_{I}\left(1-\frac{\left[\phi^{i},\left[\phi^{i},\right]\right]}{\lambda_{I}^{i}}\right) V_{\mu}$

By use of eq.(23) and recalling that

$D_{\mu} \phi^{i}=-i g\left[A_{\mu}+\Omega_{\mu}, \phi^{i}\right]$

the generalized 't Hooft tensor reads

$$
\begin{aligned}
& F_{\mu \nu}^{i}=\operatorname{Tr}\left(\phi^{i} G_{\mu \nu}\right)-\frac{i}{g} \sum_{I} \frac{1}{\lambda_{I}^{i}} \operatorname{Tr}\left(\phi^{i}\left[D_{\mu} \phi^{i}, D_{\nu} \phi^{i}\right]\right)+ \\
& +\frac{i}{g} \sum_{I \neq J} \frac{1}{\lambda_{I}^{i} \lambda_{J}^{i}} \operatorname{Tr}\left(\phi^{i}\left[\left[D_{\mu} \phi^{i}, \phi^{i}\right],\left[D_{\nu} \phi^{i}, \phi^{i}\right]\right]\right)+\ldots(25)
\end{aligned}
$$

In summary, for any gauge group $G$ we have to compute for each root $\vec{\alpha}$ the (known) commutator $\left[\phi^{i}, E^{\vec{\alpha}}\right]=\left(\vec{c}^{i} \cdot \vec{\alpha}\right) E^{\vec{\alpha}}$, where $\phi^{i}$ are the fundamental weights associated to each simple root. This gives us the set of the values of $\lambda_{I}^{i}$ to insert into eq.(25). For $S U(N)$ group $\left[\phi^{i}, E_{\vec{\alpha}}\right]=\left(\vec{c}^{i} \cdot \vec{\alpha}\right) E_{\vec{\alpha}}$ where $\left(\vec{c}^{i} \cdot \vec{\alpha}\right)=0, \pm 1$, so the projector is simply

$P^{i} V_{\mu}=\left[\phi^{i},\left[\phi^{i}, V_{\mu}\right]\right]$

and the 't Hooft tensor is the usual one

$F_{\mu \nu}^{a}=\operatorname{Tr}\left(\phi^{a} G_{\mu \nu}\right)-\frac{i}{g} \operatorname{Tr}\left(\phi^{a}\left[D_{\mu} \phi^{a}, D_{\nu} \phi^{a}\right]\right)$

For a generic group the projector is more complicated and it depends on the root chosen. For 
example in $G_{2}$ we have two 't Hooft like tensors, one for each of the two simple roots $e_{1}$ and $e_{2}$ :

$$
\begin{aligned}
& F_{\mu \nu}^{(1)}=\operatorname{Tr}\left(\phi^{(1)} G_{\mu \nu}-\frac{5 i}{4 g} \operatorname{Tr}\left(\phi^{(1)}\left[D_{\mu} \phi^{(1)}, D_{\nu} \phi^{(1)}\right]\right)\right. \\
& +\frac{i}{4 g} \operatorname{Tr}\left(\phi^{(1)}\left[\left[D_{\mu} \phi^{(1)}, \phi^{(1)}\right],\left[D_{\nu} \phi^{(1)}, \phi^{(1)}\right]\right]\right)
\end{aligned}
$$

for the breaking of the longest simple root $e_{1}$ and

$$
\begin{aligned}
& F_{\mu \nu}^{2}=\operatorname{Tr}\left(\phi^{(2)} G_{\mu \nu}\right)-\frac{49 i}{36 g} \operatorname{Tr}\left(\phi^{(2)}\left[D_{\mu} \phi^{(2)}, D_{\nu} \phi^{(2)}\right]\right) \\
& +\frac{7 i}{18 g} \operatorname{Tr}\left(\phi^{(2)}\left[\left[D_{\mu} \phi^{(2)}, \phi^{(2)}\right],\left[D_{\nu} \phi^{(2)}, \phi^{(2)}\right]\right]\right)
\end{aligned}
$$$$
-\frac{i}{36 g} \operatorname{Tr}\left(\phi^{(2)}\left[\left[\left[D_{\mu} \phi^{(2)}, \phi^{(2)}\right], \phi^{(2)}\right],\left[\left[D_{\nu} \phi^{(2)}, \phi^{(2)}\right], \phi^{(2)}\right]\right]\right)
$$

for the breaking of the second one.

\section{DISCUSSION}

The tight experimental limits on the number of free quarks in nature indicate that confinement is an absolute property, namely that the number of free quarks is strictly zero due to some symmetry. Deconfinement is then a change of symmetry.

Since color is an exact symmetry, the only way to have an extra symmetry, to be broken, is to look for a dual description of QCD. The extra degrees of freedom are infrared modes related to spatial homotopy. This is a special case of the geometric Langlands program of ref. [8].

Since the sphere at spatial infinity has dimension 2 , the relevant homotopy in $3+1$ dimensions is $\Pi_{2}$, configurations are monopoles and the quantum numbers magnetic charges.

For a generic gauge group of rank $r$ there exist $r$ different magnetic charges $Q^{a}$. The existence of nonzero magnetic charges implies a violation of Bianchi identities by the abelian gauge fields coupled to them. The gauge invariant abelian field strengths coupled to $Q^{a}$ are known as 't Hooft tensors.

Monopoles for a generic gauge group have been analyzed and the corresponding 't Hooft tensors computed in ref [12].

\section{REFERENCES}

1. L. Okun, "Leptons and Quarks", North Holland (1982).

2. Owe Philipsen, "Status of Lattice Studies of the QCD Phase Diagram", International Symposium Fundamental Problems in Hot and/or Dense QCD, Kyoto, Japan-2008, arXiv:0808.0672 [hep-ph].

3. M.D'Elia, A. Di Giacomo, C. Pica, "Two flavor QCD and confinement", Phys.Rev.D 72:114510, 2005.

4. G.Cossu, M. D'Elia, A. Di Giacomo,C. Pica, "Two flavor QCD and confinement. II", arXiv:0706.4470 [hep-lat].

5. G.'t Hooft, "Magnetic Monopole in unified gauge theories", Nucl.Phys. B 79 (1974) 276. A.M. Polyakov, "Particle spectrum in quantum field theory", JETP Lett. 20 (1974) 194.

7. M. D'Elia, A. Di Giacomo, B. Lucini, G. Paffuti, C. Pica, "Color confinement and dual superconductivity of the vacuum. IV", Phys.Rev.D71:114502,2005.

8. S.Gukov and E.Witten, "Gauge theory, ramification, and the geometric Langlands program", arXiv:hep-th/0612073.

9. A.Di Giacomo "Mechanisms of color confinement", Acta Phys.Polon. B25:215-226, 1994.

10. A. Di Giacomo, G. Paffuti, "A Disorder parameter for dual superconductivity in gauge theories", Phys.Rev.D56:6816-6823, 1997.

11. A. Di Giacomo, B. Lucini, L. Montesi, G. Paffuti, "Color confinement and dual superconductivity of the vacuum. II", Phys.Rev.D61:034504, 2000.

12. A. Di Giacomo, L. Lepori, F. Pucci, "Homotopy, monopoles and 't Hooft tensor for generic gauge groups", arXiv:0808.4041 [heplat].

13. K.Holland, P.Minkowski, M.Pepe and U.J.Wiese, "Exceptional confinement in G(2) gauge theory",Nucl.Phys. B 668 (2003) 207.

14. J. Madore, "A Classification of SU(3) Magnetic Monopoles", Commun. Math.Phys. 56 (1977) 115. 